\title{
Coqueluche : une perspective globale
}

\author{
Halperin $\mathrm{SA}^{1 *}$
}

1. Centre canadien de vaccinologie, département de pédiatrie et département de microbiologie et d'immunologie, Université Dalhousie, Centre de soins de santé IWK, Halifax (N-É)

Auteur-ressource : scott.halperin@dal.ca

Au cours des dernières années, des éclosions de coqueluche ont été signalées dans de nombreux pays, notamment aux États-Unis ${ }^{1 \text { à }}{ }^{4}$, en Australie ${ }^{5}$, au Royaume-Uni ${ }^{6}$ et au Canada. Aux États-Unis, plus de 9000 cas ont été déclarés en Californie en $2010^{7}$. En 2012, des éclosions d'envergure ont été signalées dans les États de Washington, du Minnesota et du Wisconsin. En effet, 49 États sur 50 ont déclaré un accroissement de la coqueluche en 2012 en comparaison à 2011. Seule la Californie, qui a connu une éclosion en 2010, a déclaré une diminution de l'incidence ${ }^{8}$. En Australie, l'incidence globale de la coqueluche en 2009 a augmenté à plus de 140 cas sur 100000 dépassant ainsi les taux de la plupart des autres pays industrialisés ${ }^{9}$. Au Royaume-Uni, un nombre accru de décès chez les enfants a eu lieu conjointement avec une hausse de l'incidence de la coqueluche chez les adolescents et les adultes ${ }^{6}$. Il y a d'autres pays qui n'ont pas connu de hausses de l'incidence de la coqueluche, notamment la France, le Danemark et la Suède, et certains autres pays européens continuent de déclarer des taux de la coqueluche faibles. Comme il est mentionné dans ce numéro, au Canada, une activité accrue de la coqueluche a été signalée dans quelques provinces ou régions ${ }^{10,11}$.

Y a-t-il une résurgence de la coqueluche? La coqueluche est une maladie cyclique atteignant un sommet tous les deux à cinq ans. Est-ce simplement un sommet cyclique attendu? La surveillance s'est-elle améliorée ou y a-t-il eu des changements aux tests diagnostiques? L'organisme est-il devenu plus virulent? Le vaccin est-il devenu moins efficace? Comment ces changements se répercutent-ils sur la situation canadienne?

L'épidémiologie de la coqueluche dans le monde peut être le résultat de nombreux facteurs avec une influence qui varie dans chaque pays. Aux États-Unis, l'éclosion de la Californie en 2010, qui a entraîné le décès de dix enfants, était frappante parce qu'elle concernait des enfants d'âge scolaire de 7 à 10 ans. Un laps de temps important depuis l'administration de la dose vaccinale anticoquelucheuse préscolaire a été un facteur de risque qui suppose un affaiblissement de l'immunité induite par le vaccin. Un nombre appréciable d'enfants non vaccinés peut aussi avoir contribué à l'éclosion ${ }^{2}$. La durée de la protection était sensiblement plus courte chez les enfants qui avaient reçu tous leurs vaccins anticoquelucheux au moyen d'un vaccin anticoquelucheux acellulaire ${ }^{1,4}$. En 2012, les États de Washington, du Wisconsin et du Minnesota ont aussi connu un sommet de l'incidence de la coqueluche chez les enfants d'âge scolaire. Dans l'État de Washington, une durée de protection courte après l'administration d'un vaccin anticoquelucheux acellulaire (dcaT) à contenu réduit chez les préadolescents a également été un facteur ${ }^{12}$. Donc, les éclosions aux États-Unis semblent associées à une durée de protection plus courte que prévu liée aux vaccins anticoquelucheux acellulaires, à l'administration de tous les vaccins anticoquelucheux au moyen de vaccins acellulaires et à des poches d'enfants totalement non vaccinés. En Australie, on a signalé une augmentation de cas chez les enfants d'âge préscolaire à cause de l'élimination de la dose de rappel du vaccin combiné contre la diphtérie, la coqueluche acellulaire et le tétanos (DCaT) dans la deuxième année de vie ${ }^{5,9}$. Des taux élevés de coqueluche ont aussi été signalés chez les adultes, peut-être en raison d'une grande disponibilité d'un test sérologique de la coqueluche utilisé depuis les années $1990^{9}$. En Angleterre, une éclosion de la coqueluche a été associée à un nombre accru de décès chez les enfants. L'éclosion peut être attribuable à l'absence d'une dose de rappel chez les adolescents ${ }^{6,13}$.

Compte tenu des données récentes qui associent une dose unique du vaccin anticoquelucheux à germes entiers à une plus grande durée de protection, on peut supposer que la dose ou les doses de vaccin anticoquelucheux à germes entiers ont précédé les doses de vaccin anticoquelucheux acellulaire, car les vaccins anticoquelucheux

acellulaires ont totalement remplacé les vaccins anticoquelucheux à germes entiers aux États-Unis. Il est difficile d'imaginer qu'une protection directe découlant de la dose ou des doses initiales demeure. Au lieu, il se peut que la meilleure protection soit celle où la dose initiale du vaccin dirige le système immunitaire vers une réponse de 
type $T h 1^{14}$. Le vaccin anticoquelucheux acellulaire, avec son adjuvant à base d'aluminium, fait dévier le système immunitaire vers une réponse biaisée de type Th2. En revanche, même s'il est aussi adjuvanté avec de l'aluminium, le vaccin anticoquelucheux à germes entiers engendre une réponse biaisée de type Th1, semblable à une infection naturelle, qui peut être liée à une présence résiduelle de lipopolysaccharide (endotoxine) de $B$. pertussis, un puissant adjuvant Th1. En dépit de ces raisons immunologiques marquant la supériorité des vaccins anticoquelucheux à germes entiers sur les vaccins acellulaires, il y a eu un nombre accru de déclarations de l'activité de la coqueluche dans les pays qui utilisent un vaccin anticoquelucheux à germes entiers, notamment au Brésil et au Chili ${ }^{15}$. Néanmoins, il n'y a pas qu'un seul vaccin anticoquelucheux à germes entiers, il en existe plusieurs, et aucune corrélation n'existe entre leur efficacité relative et les essais intracérébraux utilisés chez les souris comme exigence réglementaire pour leur approbation. Même si des données des essais cliniques de grande envergure menés sur les vaccins anticoquelucheux acellulaires dans les années 1990 laissent entendre que l'efficacité d'un " bon » vaccin anticoquelucheux à germes entiers est supérieure à celle des vaccins anticoquelucheux acellulaires, le vaccin anticoquelucheux à germes entiers utilisé dans deux des plus grands essais comparatifs randomisés était très inférieur à tous les vaccins anticoquelucheux acellulaires testés ${ }^{16}$. De plus amples renseignements sont nécessaires pour déterminer si les autres pays qui utilisent les mêmes vaccins anticoquelucheux à germes entiers ont connu ces mêmes hausses.

Des inquiétudes ont été soulevées par rapport au fait que les changements dans le Bordetella pertussis puissent aussi contribuer à un plus grand nombre de cas. Des souches ayant une production accrue de toxine coquelucheuse ont été isolées, et les souches qui expriment une pertactine altérée et, même les souches négatives pour la pertactine, sont de plus en plus communes ${ }^{17,18}$. Certaines personnes ont laissé entendre que ces souches resurgissent sous la pression immunologique émanant des vaccins anticoquelucheux acellulaires actuels. Malgré l'isolement grandissant de ces souches, il n'y a aucune preuve claire qu'elles sont associées à une plus grande virulence clinique ou qu'elles sont la cause d'une activité accrue de la coqueluche ${ }^{19}$.

Des éclosions ont également été signalées dans plusieurs provinces canadiennes. En Saskatchewan, en 2010, l'incidence de la coqueluche chez les enfants de moins d'un an a radicalement augmenté. Six décès ont été signalés. Les taux dépassaient ceux déclarés en Californie pour la même année. La sous-vaccination, notamment chez certaines collectivités des Premières Nations, peut avoir contribué à l'augmentation des taux de coqueluche. En 2012, le Nouveau-Brunswick a déclaré plus de 1400 cas de coqueluche, ce qui représente près d'un tiers des cas signalés au Canada ${ }^{10}$, avec une incidence qui dépassait les taux déclarés de l'État de Washington, du Wisconsin et du Minnesota. Comparativement à ce qui s'est passé en Saskatchewan, les cas au Nouveau-Brunswick étaient surtout axés sur les enfants d'âge scolaire. Très peu d'hospitalisations et aucun décès chez les enfants n'ont eu lieu. Même si une analyse complète de l'éclosion du Nouveau-Brunswick n'a pas encore été présentée, une diminution de la durée de protection après l'administration de la dose vaccinale anticoquelucheuse préscolaire (mais pas la dose de vaccin dCaT chez les adolescents), comme il a été signalé aux États-Unis, a pu être un facteur. En Colombie-Britannique, les régies régionales de la santé de Fraser et de Coastal (région de Vancouver) ont aussi reçu un nombre croissant de déclarations de la coqueluche. Aucune augmentation n'a été signalée dans les autres endroits de la province. La cause de la résurgence localisée de la coqueluche en Colombie-Britannique n'est pas claire. Une évaluation plus poussée des taux de refus de vaccination dans la région de Vancouver en comparaison avec d'autres endroits de la province pourrait être révélatrice. En Ontario, une éclosion localisée dans la partie sud-ouest de la province est nettement associée à une collectivité qui refuse la vaccination des enfants ${ }^{11}$.

Comme il a été observé ailleurs dans le monde, l'épidémiologie de la coqueluche au Canada semble être le résultat d'une combinaison de facteurs, notamment les cycles naturels de deux à cinq ans, l'omission de se faire vacciner et l'échec vaccinal (tant l'échec primaire lié à une efficacité du vaccin plus faible après la vaccination que l'échec secondaire attribuable à un affaiblissement de l'immunité). Quelles sont les répercussions pour le Canada? Même si jusqu'ici, il n'y a pas de preuve définitive que les modifications de l'organisme entraînent une résurgence de la maladie, il est important de continuer la surveillance à ce chapitre au Canada et dans le monde. L'éloignement par rapport à un diagnostic basé sur la culture en faveur d'un diagnostic basé sur la réaction de polymérisation en chaîne indique que la surveillance des modifications dans l'organisme sera plus difficile. Pour que la surveillance des souches se poursuive, les laboratoires de diagnostic doivent collaborer afin de prélever une proportion d'écouvillons nasopharyngiens soumis dans le cadre du diagnostic de la coqueluche ou doivent 
conserver des sécrétions résiduelles pour un isolement ultérieur des organismes aux fins de surveillance microbiologique. La surveillance continue des cohortes canadiennes qui ont reçu toutes leurs doses de vaccin acellulaire est aussi importante pour explorer davantage la durée de protection découlant du vaccin anticoquelucheux acellulaire. Des données plus précises sont requises sur la couverture vaccinale à l'échelle locale et provinciale afin de cerner les poches de sous-vaccination. II est essentiel de mettre au point de nouveaux vaccins qui sont plus efficaces avec une durée de protection plus longue. Les nouvelles technologies, telles que les vaccins anticoquelucheux vivants atténués ${ }^{20}$ et les vaccins avec de nouveaux adjuvants qui dirigent le système immunitaire vers une réponse du type $T h 1^{21}$, peuvent apporter la solution, mais on est loin d'être là. Jusqu'à ce que de meilleurs vaccins soient disponibles, il faudra faire un usage optimal des vaccins actuels. Une couverture vaccinale élevée et à temps est encore la meilleure méthode de lutte contre la coqueluche au Canada.

\section{Références}

1. Klein NP, Bartlett $\mathrm{J}$, Rowhani-Rahbar A, et al. Waning protection after fifth dose of acellular pertussis vaccine in children. N Eng J Med. 2012;367:1012-19.

2. Misegades LK, Winter K, Harriman K, Talarico J, Messonnier NE, Clark TA, Martin SW. Association of childhood pertussis with receipt of 5 doses of pertussis vaccine by time since last vaccine dose, California, 2010. JAMA. 2012;308:2126-32.

3. Washington State Department of Health. 2012 Pertussis Summary for Washington State. 2013; http://www.doh.wa.gov/YouandYourFamily/lllnessandDisease/WhoopingCough.asp

4. Witt MA, Arias L, Katz PH, Truong ET, Witt DJ. Reduced risk of pertussis among persons ever vaccinated with whole cell pertussis vaccine compared to recipients of acellular pertussis vaccines in a large US cohort. Clin Infect Dis. 2013;56:1248-54.

5. Campbell P, McIntyre P, Quinn H, Hueston L, Gilbert GL, McVernon J. Increased population prevalence of low pertussis toxin antibody levels in young children preceding a record pertussis epidemic in Australia. PLoS One. 2012;7:e35874.

6. Amirthalingam G. Strategies to control pertussis in infants. Arch Dis Child. 2013;98:552-5. http://www.cps.ca/en/impact

7. California Department of Public Health. Pertussis (whooping cough). http://www.cdph.ca.gov/healthinfo/discond/pages/pertussis.aspx

8. Centers for Disease Control and Prention. Pertussis outbreak trends. http://www.cdc.gov/pertussis/outbreaks/trends.html

9. MacIntyre P. Is Australia the pertussis capital of the world. National Pertussis Workshop. 25-26 August 2011, Sydney.

http://www.ncirs.edu.au/news/archive.php

10. Smith T, Lourenco J, Desai S, Deehan H. Surveillance de la coqueluche au Canada: tendances jusqu'à 2012, RMTC 2014-40-3.

http://www.phac-aspc.gc.ca/publicat/ccdr-rmtc/14vol40/dr-rm40-03/dr-rm40-03-per-fra.php

11. Deeks SL, Lim GH, Walton R, Fediurek J, Lam F, Walker C, Walters J, Alexander D, Crowcroft NS. Prolonged pertussis outbreak in Ontario originating in an under-immunized religious community. RMTC 201440-3.

12. Acosta A, DeBolt C, Tasslimi A, Lewis M, Stewart L, Misegades L, Messonier N, Clark T, Martin S, Patel M. Vaccine effectiveness and duration of protection of tetanus toxoid, reduced diphtheria toxoid and acellular pertussis among adolescents, Washington State, 2012. Presented at the 10th International Symposium on Bordetella, 8-11 September 2013. Abstract O7.

13. Campbell H, Amirthalingam G, Andrews N, Fry NK, George RC, Harrison TG, Miller E. Accelerating control of pertussis in England and Wales. Emerg Infect Dis. 2012;18:38-47. 
14. Ross PJ, Sutton CE, Higgins S, Allen AC, Walsh K, Misiak A, Lavelle EC, McLoughlin RM, Mills KH. Relative contribution of Th1 and Th17 cells in adaptive immunity to Bordetella pertussis: towards the rational design of an improved acellular pertussis vaccine. PLoS Pathog. 2013;9:e1003264.

15. Miranda C, Porte L, García P. Bordetella holmesii in nasopharyngeal samples from Chilean patients with suspected Bordetella pertussis infection. J Clin Microbiol. 2012;50:1505.

16. Cherry JD (2012) Why do pertussis vaccines fail? Pediatrics. 129:968-70.

17. King AJ, van der Lee S, Mohangoo A, van Gent M, van der Ark A, van de Waterbeemd B. Genome-wide gene expression analysis of Bordetella pertussis isolates associated with a resurgence in pertussis: elucidation of factors involved in the increased fitness of epidemic strains. PLoS One. 2013;8(6):e66150.

18. Queenan AM, Cassiday PK, Evangelista A. Pertactin-negative variants of Bordetella pertussis in the United States. N Engl J Med. 2013;368:583-4.

19. Bodilis $\mathrm{H}$, Guiso N. Virulence of pertactin-negative Bordetella pertussis isolates from infants, France. Emerg Infect Dis. 2013;19:471-4.

20. Kammoun H, Roux X, Raze D, Debrie AS, De Filette M, Ysenbaert T, Mielcarek N, Saelens X, Fiers W, Locht C. Immunogenicity of live attenuated B. pertussis BPZE1 producing the universal influenza vaccine candidate M2e. PLoS One. 2013;8:e59198.

21. Polewicz M, Gracia A, Garlapati S, van Kessel J, Strom S, Halperin SA, Hancock RE, Potter AA, Babiuk LA, Gerdts V. Novel vaccine formulations against pertussis offer earlier onset of immunity and provide protection in the presence of maternal antibodies. Vaccine. 2013;31:3148-55. 